\title{
Chinese expert consensus on chrono-chemotherapy for oral squamous cell carcinoma
}

\author{
Kai Yang" ${ }^{1 \#}$, Wei Guo ${ }^{2 \#}$, Moyi Sun ${ }^{3}$, Jiawei Zheng ${ }^{2}$, Longjiang Li $^{4}$, Zhangui Tang ${ }^{5}$, Guoxin Ren ${ }^{2}$, \\ Dan Chen ${ }^{1}$, Xiaoqiang Lv ${ }^{1}$, Jie Zhang ${ }^{6}$, Zhengxue Han $^{7}$, Qing Xi ${ }^{8}$, Zhijun Sun ${ }^{9}$, Xiaopin Chen ${ }^{10}$, \\ Tao Zhang ${ }^{10}$, Zonggang Che ${ }^{11}$, Wei Ran ${ }^{12}$, Jian Meng ${ }^{13}$, Guilin Huang ${ }^{14}$, Hong Tang ${ }^{1}$, Can Xiao ${ }^{15}$
}

${ }^{1}$ Department of Oral and Maxillofacial Surgery, The First Affiliated Hospital of Chongqing Medical University, Chongqing 400016, China; ${ }^{2}$ Department of Oromaxillofacial Head and Neck Oncology, Shanghai Ninth People's Hospital, College of Stomatology, Shanghai Jiao Tong University School of Medicine, Shanghai 200011, China; ${ }^{3}$ Department of Oral and Maxillofacial Surgery, The Third Affiliated Hospital, Air Force Medical University (School of Stomatology, Fourth Military Medical University), Xi'an710032, China; ${ }^{4}$ Department of Head and Neck Oncology Surgery, West China Hospital of Stomatology, Sichuan University, Chengdu 610041, China; ${ }^{5}$ Department of Oral and Maxillofacial Surgery, Xiangya School of Stomatology, Central South University, Changsha 410013, China; ' Department of Oral and Maxillofacial Surgery, School and Hospital of Stomatology, Peking University, Beijing 100081, China; ${ }^{7}$ Department of Oral and Maxillofacial-Head and Neck Surgery, Beijing Stomatological hospital, Capital Medical University, Beijing 100050, China; ${ }^{8}$ Oral Department of 1st Medical Center, Chinese PLA General Hospital, Beijing 100853, China; ${ }^{9}$ Department of Oral and Maxillofacial-Head Neck Oncology Surgery, School and Hospital of Stomatology, Wuhan University, Wuhan 430079, China; ${ }^{10}$ Oncology Department, The First Affiliated Hospital of Chongqing Medical University, Chongqing 400016, China; ${ }^{11}$ Department of Oral and Maxillofacial Surgery, Zibo Central Hospital, Zibo 255036, China; ${ }^{12}$ Department of Oral and Maxillofacial Surgery, First Affiliated Hospital of Sun Yat-sen University, Guangzhou 510080, China; ${ }^{13}$ Department of Oral and Maxillofacial Surgery, Xuzhou Central Hospital, Xuzhou 221009, China; ${ }^{14}$ Department of Oral and Maxillofacial Surgery, Stomatological Hospital, Zunyi Medical University, Zunyi 563000, China; ${ }^{15}$ Department of stomatology, The First Affiliated Hospital of Soochow University, Suzhou 215006, China

\#These authors contributed equally to this work.

Correspondence to: Wei Guo. 639 Manufacturing Bureau Road, Shanghai 200011, China. Email: guoweicn@yahoo.com; Kai Yang. 1 Youyi Road, Yuzhong, Chongqing 400016, China. Email: cqfyyk@hotmail.com.

\begin{abstract}
Chemotherapy is one of the primary methods for the treatment of oral squamous cell carcinoma, and it is also an essential part of the comprehensive treatment of oral squamous cell carcinoma. However, the "double-edged sword" effect of chemotherapeutic drugs in killing both cancer cells and normal cells in vivo has restricted its clinical application to a certain extent. Chrono-chemotherapy based on human circadian rhythm is safer and more effective than traditional chemotherapy, which has been confirmed by a large number of studies. In order to promote and standardize the clinical application of this technology, we organized relevant experts to discuss and formulate the Chinese expert consensus. The consensus provides standardized suggestions on the related concepts, principles, drug selection, administration time, and methods of chrono-chemotherapy for oral squamous cell carcinoma, which can provide a reference for clinical practice.
\end{abstract}

Keywords: Oral squamous cell carcinoma (OSCC); oral cancer; chrono-chemotherapy; circadian rhythm; circadian clock; expert consensus

Received: 25 June 2019; Accepted: 24 October 2019; Published: 28 November 2019.

doi: 10.21037/fomm.2019.11.01

View this article at: http://dx.doi.org/10.21037/fomm.2019.11.01 
Oral cancer includes cancer occurring in the buccal mucosa, the floor of the oral, $2 / 3$ in front of the tongue, alveolar ridge, post-molar triangle, and hard palate $(1,2)$. Oral squamous cell carcinoma (OSCC) accounts for more than $90 \%$ of oral cancer (1). Chemotherapy is one of the main treatments for OSCC and is also an essential component of comprehensive treatment of OSCC (2-4). However, when chemotherapy drugs kill cancer cells in the body, they also have a destructive effect on normal human cells, especially on actively proliferating bone marrow hematopoietic cells, intestinal epithelial cells, hepatocyte, and nephrocyte, causing adverse reactions such as bone marrow suppression, nausea, vomiting, liver, and kidney dysfunction. The double-edged sword effect of chemotherapy drugs limits its clinical application. The chrono-chemotherapy developed according to the circadian rhythm and the circadian clock has the advantage of reducing the adverse reactions of chemotherapy drugs and improving the therapeutic effect, which has gradually been clinically confirmed (5-8). It is getting increasing attention (9-11).

The current research (12-15) proves that the proliferation, cycle distribution, DNA synthesis, molecular targets and drug metabolism enzyme activity of normal human cells and tumor cells have circadian rhythm fluctuations, and their fluctuation variation difference often reaches several times. Therefore, the circadian rhythm has an essential influence on the pharmacokinetics and pharmacodynamics of chemotherapeutic drugs. Animal experiments and clinical studies $(13,14)$ have also demonstrated that there are over 40 chemotherapeutic drugs whose efficacy and adverse effects are significantly different when they are delivered at different timepoints within 24 hours. For the same individual, the drug is delivered at different time within the 24-hour timescale, the difference on efficacy can be more than 2 times, and the difference on adverse reactions can be as much as 10 times. Chrono-chemotherapy is based on the law of fluctuation of circadian rhythms, such as cell proliferation, cell cycle, DNA synthesis, drug metabolism enzyme activity and target of the body, and at the same time combined with the pharmacological mechanism of the drugs. Chrono-chemotherapy is to optimize the drug delivery time depending on comprehensive optimization, to maximize the anti-cancer effect, minimize adverse reactions (14), and improve the quality of life and survival of patients.

A large number of studies has confirmed that chronochemotherapy is safer and more effective than conventional chemotherapy (13-17), but the chrono-chemotherapy is still far from being popularized in China; meanwhile, there is no expert consensus or clinical application guidelines of chrono-chemotherapy for any cancer, including OSCC. Therefore, there is crucial clinical significance to formulate an expert consensus on chrono-chemotherapy for OSCC timely. Given this, based on the reference to the domestic and international research on the chrono-chemotherapy related research, as well as a combination with the current medical situation in China, we organized relevant experts to discuss and formulate the expert consensus on the clinical application of chrono-chemotherapy for OSCC. We are aiming at promoting and standardizing chronochemotherapy for OSCC and creating the benefit of the majority of patients with OSCC.

\section{Several important concepts of chrono- chemotherapy}

\section{Circadian rhythm}

More than 300 life activities are exhibiting a circadian fluctuation of approximately 24 hours in mammals and humans, such as rest-activity, body temperature, heart rate, blood pressure, cell proliferation, synthesis and repair of DNA, hormone secretion, and immune response. It is called the circadian rhythm $(10,18)$. The circadian rhythm is an endogenous adaptation mechanism, which is a long-term evolution of the biology on Earth to adapt to the living environment of the Earth's rotation. It is the inherent basic feature and premise of life activities and still exists even in the absence of light $(9,10,18)$. The circadian rhythm not only leads to rhythmic changes in the biological behavior but also rhythmic changes in the cellular and molecular levels $(5,11)$. Since the biological rhythm of an organism is easily interfered by various factors, and in order to eliminate these interferences, it is necessary to divide 24 hours into several time points (generally 6) when we determine whether a life activity shows a circadian rhythm. The fluctuation value of each time point was detected, and the cosine test showed that the day and night fluctuations coincide with the cosine function law $(\mathrm{P}<0.05)$, which means that the organism exhibit the circadian rhythm (18).

\section{The circadian clock, circadian clock gene, and clock- controlled genes (CCGs)}

The circadian clock is an endogenous time-regulating system formed by the long-term evolution of the biology on the earth to adapt to the rotation of the earth. Its core is 
the rhythmic expression of the circadian clock gene $(7,9,10)$. The circadian clock gene is present in almost all cells in the human $(10,19)$, and 14 circadian clock genes have been discovered so far, namely CLOCK, BMAL1, PER1, PER2, PER3, DEC1, DEC2, CRY1, CRY2, TIM, CKIE, ROR $\alpha$, $N P A S 2$ and $R E V-E R B \alpha$. The interaction between the circadian clock genes forms multiple positive and negative feedback loops, thus showing a periodic rhythm expression of about 24 hours $(7,18)$. In mammals, the clock gene regulates approximately $43 \%$ protein-coding genes in the genome, and these genes regulated by the clock gene are called CCGs $(10,20)$. CCGs also exhibit a circadian rhythm of about 24 hours. CCGs are involved in the regulation of various aspects of life activity, including cell cycle, proliferation, cell metabolism, and secretion, which is the molecular basis of circadian rhythms of life activities.

\section{Activity phase and rest phase}

The time of circadian rhythm and chrono-chemotherapy is often expressed as phase (11). The activity phase and rest phase are vital concepts to understand the time changes in circadian rhythm and chrono-chemotherapy. The circadian rhythm within the 24-hour timescale is divided into 12-hour activity phase and 12 -hour rest phase $(11,14)$. The activity phase and rest phase can be further divided into three phases: early phase, middle phase, and late phase (4 hours per each phase). For humans, the activity phase means daytime, and rest phase means nighttime. Due to the particularity of circadian rhythms and chrono-chemotherapy, many findings were obtained from rodents. It is currently believed that the experimental results obtained from rodents can be inferred to humans $(14,21)$, but rodents are nocturnal animals, and their rest phase and activity phase are opposite to humans. Therefore, when the experimental results obtained from rodents are extrapolated to human beings, they should be judged from the concept of rest phase and activity phase, that is, the rodents are in the rest phase or the activity phase, and their circadian rhythm is highly correlated with the corresponding rest phase or activity phase of human beings. The time of the circadian rhythm of the rodent is defined by hours after light onset (HALO) or zeitgeber time (ZT), rather than clock time. Zero HALO is the light-on time, 12 HALO is the light-off time, that is before 12 HALO is the rest phase, and after 12 HALO is the activity phase (14). The understanding of the above phases is the basis for mutual reference and verification of the results of human and mouse chrono-chemotherapy.

\section{Mechanism of chrono-chemotherapy}

\section{Differences in circadian rhythms between tumor cells and normal tissue cells}

There is circadian rhythm in activities of that the growth, proliferation, and DNA synthesis of normal human cells and tumor cells, but the circadian rhythm of tumor cell growth and proliferation is different from that of normal cells $(7,13)$. The peak of proliferation, DNA synthesis, and the proportions of S- and G2/M-phase cells of human tumor cells occurs mostly in the resting phase, especially in the early and middle stages of the resting phase, while that of normal cells of bone marrow and intestinal mucosal epithelium appears in the early and middle active phase $(7,12-14)$. Generally, there is a time difference of 8-12 hours between the occurrence of the peak of proliferation, DNA synthesis, and the proportions of S- and G2/M-phase cells of human tumor cells and that of normal cells (bone marrow, intestinal mucosal epithelium, and so on). Since cells are more sensitive to chemotherapeutic drugs when they proliferate and DNA synthesis actively, delivering chemotherapeutic drugs at the time when normal tissue cells are best tolerated (least sensitive) and tumor cells are least tolerated (most sensitive) can achieve the purpose of increasing the efficacy of drugs and reducing adverse drug reactions. Current studies have also approved that the peak of the proliferation of OSCC occurs at $6.60 \mathrm{HALO}$ in the middle of the resting phase, and the trough of it occurs in the middle of the active phase of 18.16 HALO (22).

\section{Effects of circadian rhythms on pharmacokinetics and pharmacodynamics}

The activity of some critical metabolic enzymes for the drug in the body exhibits circadian rhythm fluctuations, and there are often several times differences between the peaks and troughs of fluctuations $(7,12,14)$. Therefore, the processes of bioavailability, blood concentration, transportation, metabolism, and excretion of drugs in the body are affected by the circadian rhythm of the body. There are the most significant medicines whose metabolic enzyme activity exhibiting the circadian rhythm as follows: dihydropyrimidine dehydrogenase (DPD) is a crucial enzyme for the metabolism of 5-fluorouracil (5-FU), whose activity is significantly higher in resting phase than inactive phase both in healthy people and cancer patients; and its activity changes exhibit circadian rhythm. The DPD activity in the human increases by more than 40\% from 00:00 to 10:00 compared with other times, and its activity peaks occur at around 04:00, the amplitude 
of fluctuations during day and night is 3 times. However, the proportions of $\mathrm{S}$ and G2/M phase cells of human intestinal mucosa, skin and bone marrow normal cells is the lowest during this period, and the tolerant to drugs during this period is better, so delivering drug during this period can reduce toxicity and increase efficiency $(7,12)$. Glutathione (GSH) is an essential physiological antioxidant in the human body. It participates in the detoxification process of cells in the body and can reverse the toxicity of platinum, alkylating agents and anthracyclines to normal cells in vivo. It can protect normal cells from oxidative damage without reducing the anticancer activity of the drug. The secretion of GSH also shows circadian rhythm, and the secretion difference at different times of day and night can be more than 3 times in the same individual. The peak of GSH is at often around 16:00 in the human body $(7,14)$, and the drug delivery during this period can reduce the adverse reactions of platinum, alkylating agents and anthracyclines. Thymidylate synthetase (TS) is a target of 5-FU and methotrexate, and it is a crucial target for adverse reactions. The activity of TS also shows circadian rhythm, and its activity of normal human cells is significantly lower in the resting phase than in the active phase, with a trough between 00:00 and 04:00 (12). Therefore, administration of medicine in resting phase can significantly reduce the occurrence of adverse reactions.

\section{Blood flow of solid tumor blood flow exhibits circadian rbythm}

The blood flow of solid tumors and normal organs shows circadian rhythm change and the blood flow of solid tumors is significantly higher in the resting phase than that in the active phase. In the middle of the resting phase (peak at about 17 HALO), the blood flow of the tumor is highest, while the circadian rhythm of the blood flow of normal organs such as liver, lung, and kidney is roughly opposite to that of the tumor $(23,24)$. The circadian rhythm of blood flow in tumors and normal organs will affect the local concentration of the medicine, thereby affecting the efficacy and adverse reactions of medicine delivered at different times in the day and night.

\section{Basis of chrono-chemotherapy for OSCC}

\section{The pharmacological basis of chrono-chemotherapy for OSCC}

Cisplatin, carboplatin, paclitaxel, docetaxel, and 5-FU are the first-line choice for the treatment of $\operatorname{OSCC}(2,3)$. Moreover, current studies have shown that all of them show the characteristic of chrono-pharmacology $(7,12-17)$, so they are suitable for the chrono-chemotherapy of OSCC.

\section{The clinical basis of chrono-chemotherapy for OSCC}

Clinical studies have shown that chrono-chemotherapy for OSCC can reduce adverse drug reactions and increase drug efficacy $(15,16,24,25)$. Seventy-three patients with T3 and T4 OSCC were randomized into the chronochemotherapy group and the conventional chemotherapy group in the study by Yang et al. (16). The chemotherapy drugs were carboplatin and 5-FU (CF regimen) in their study. In the chrono-chemotherapy group, the carboplatin and 5-FU delivery time were 16:00-18:00, and 22:00-07:00 next day, respectively; the conventional chemotherapy group was administered in the morning, ending before getting off work. Two groups of patients underwent 2 chemotherapy cycles. The results showed that the total effective rate (objective response rate) of the chronochemotherapy group was significantly higher than that of the conventional chemotherapy group $(75.68 \%$ versus $52.78 \%$ ), and the incidence of adverse reactions was significantly reduced $(24.32 \%$ versus $58.34 \%)$. Neoadjuvant chrono-chemotherapy for 43 elderly patients with T3 and T4 OSCC with the same CF regimen and delivery time also demonstrated that chrono-chemotherapy significantly increased the objective response rate $(69.57 \%$ versus $45.00 \%$ ), and reduced the incidence of adverse reactions (34.78\% versus $65.00 \%$ ) (25). In another study of palliative chemotherapy with the combination of paclitaxel, carboplatin, and 5-FU for 49 patients with recurrent and metastatic oral and oropharyngeal squamous cell carcinoma, 49 patients were randomized into a chronochemotherapy group and a conventional chemotherapy group. The intravenous delivery time of paclitaxel, carboplatin and 5-FU was 03:00-05:00, 16:00-20:00, and 22:00-07:00 next day, respectively. The drug delivery in conventional chemotherapy group was completed during normal working hours. The results showed that the objective response rate of chrono-chemotherapy was significantly higher than that of conventional chemotherapy (71.43\% versus $42.86 \%)$.

Moreover, the overall median survival time was significantly prolonged (15.3 versus 10.6 months). Also, the incidence rate of adverse reactions was significantly reduced ( $46.43 \%$ versus $76.19 \%$ ), especially the incidence rate of 
grade III to IV severe adverse reactions is significantly reduced (7.14\% versus $33.33 \%$ ) (17). Tsuchiya et al. (26) compared the adverse reactions of chrono-chemotherapy and conventional chemotherapy with a combination of docetaxel, cisplatin, and 5-FU for patients with stage III or IV OSCC. Docetaxel and cisplatin were delivered at night (rest phase) in the chrono-chemotherapy group, while docetaxel and cisplatin were delivered in the morning (active phase) in the conventional chemotherapy group. The self-pairing cross design experiment proved that chronochemotherapy significantly reduced the incidence of adverse reactions. Also, the effect of chrono-chemotherapy on increasing drug efficacy and reducing adverse drug reactions has been confirmed in the clinical treatment of various malignant tumors, such as colorectal cancer, gastric cancer and lung cancer $(6,7,11-15)$. Therefore, chronochemotherapy is an effective chemotherapy method worth promoting.

\section{Indications and contraindications for chrono- chemotherapy for OSCC}

\section{Indications}

(I) Neoadjuvant chemotherapy before surgical resection and postoperative adjuvant chemotherapy in locally advanced patients;

(II) Palliative treatment of metastatic or (and) recurrent and unresectable OSCC;

(III) Comprehensive therapy of combined radiotherapy, targeted therapy, and biological therapy.

\section{Contraindications}

(I) Severe heart failure, liver failure, kidney failure, lung failure, and other critical organ failures;

(II) Severe myelosuppression, coagulation disorders, which are unrecoverable;

(III) Hypertension, diabetes, neuropsychiatric, or immune system diseases, which are not well controlled;

(IV) Pregnant or lactation women;

(V) Those who are allergic to platinum or paclitaxel or 5-FU drugs;

(VI) Eastern Cooperative Oncology Group (ECOG) physical status scores $\geq 3$ or Karnofsky (KPS) behavior status score $<70$ points;

(VII) Expected survival time $<3$ months;

(VIII) Other patients who are not suitable for chemotherapy.

\section{Recommended drug, method and time of drug delivery by chrono-chemotherapy for OSCC}

\section{Recommended drugs and regimens}

Drugs used for chrono-chemotherapy for OSCC need to meet two characteristics. The first is that it has been clinically proven to be an effective drug for the treatment of OSCC, and the second is that the drug must have the characteristics of chronopharmacology. Based on the above principles, the expert group recommended cisplatin $\left(80-120 \mathrm{mg} / \mathrm{m}^{2} / \mathrm{d}\right)$, carboplatin (300-400 $\left.\mathrm{mg} / \mathrm{m}^{2} / \mathrm{d}\right)$, paclitaxel $\left(135-175 \mathrm{mg} / \mathrm{m}^{2} / \mathrm{d}\right)$, docetaxel $\left(70-75 \mathrm{mg} / \mathrm{m}^{2} / \mathrm{d}\right)$ and $5-\mathrm{FU}\left(500-750 \mathrm{mg} / \mathrm{m}^{2} / \mathrm{d}\right)$ as chronochemotherapy drugs for OSCC currently. The recommended combination chemotherapy regimens are: cisplatin $+5-\mathrm{FU}$ (PF regimen), carboplatin $+5-\mathrm{FU}$ (CF regimen), docetaxel + cisplatin + 5-FU (TPF regimen), paclitaxel + cisplatin + $5-\mathrm{FU}$ (PCF regimen). The above regimens can be selected according to the clinical situation (2). However, TPF or PCF regimen is preferred for neoadjuvant chemotherapy and sequential chemotherapy, and PF or CF regimen is preferred for combined radiotherapy or targeted therapy.

\section{Method of drug delivery}

There are two methods of drug delivery of chronochemotherapy currently: constant-rate infusion and sinusoidal rate infusion. The method of constant-rate infusion is to set a start time and a completion time during which the drug is delivered at a constant velocity. A multi-channel programmable infusion pump is used for sinusoidal flow rate administration method. Start time and completion time are also set, but in this specific period, a peak flow rate time point is also set at the same time. That is, the velocity of drug delivery is sinusoid gradually increased from the start time, and after reaching the peak flow time point, the velocity gradually decreases symmetrically to the completion time. Both methods of drug delivery can achieve the purpose of chrono-chemotherapy for OSCC. Therefore, the expert group recommended that either of the two administration methods can be selected according to the situation of their medical institutions. However, sinusoidal rate infusion schedule is more consistent with the change rule of the circadian rhythm in the body, and it can accurately control the peak time of the drug. Therefore, it is recommended first.

\section{The time of drug delivery}

The drug delivery time is expressed by the time scale of 


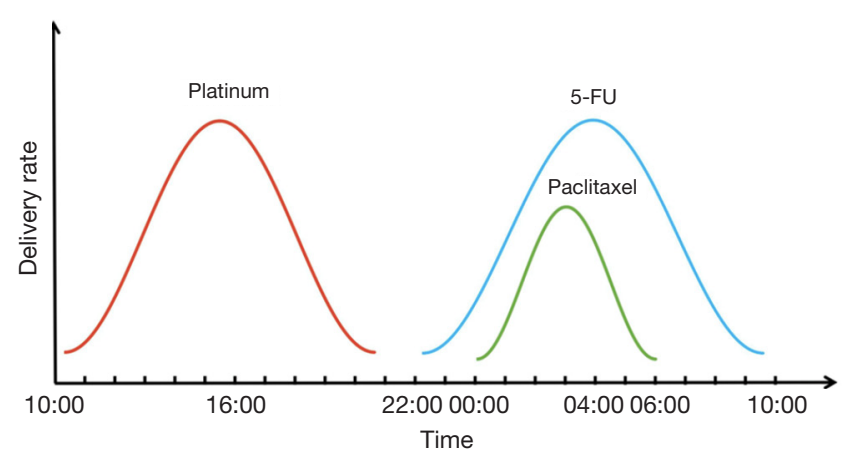

Figure 1 Time and method of sinusoidal curve flow rate method for drug delivery. 5-FU, 5-fluorouracil.

24 hours per day, and the recommended drug delivery time for each drug is as follows.

(I) Platinum drugs include cisplatin and carboplatin, with the same chronological action mode and the same delivery time. Constant-rate infusion schedule: start time is $16: 00$, and the completed time is 20:00. Sinusoidal rate infusion schedule: start time is $10: 15$, completed time is $21: 45$, peak flow time is 16:00 (Figure 1);

(II) Constant-rate infusion schedule for 5-FU: the start time is 22:00, and the completed time is 07:00 the next day. Sinusoidal rate infusion schedule: start time is $22: 15$, completed time is $09: 45$ next day, and peak flow time is 04:00 next day (Figure 1);

(III) Paclitaxel includes paclitaxel and docetaxel, with the same chronological action mode and the same delivery time. Constant-rate infusion schedule: start time is $03: 00$, and the completion time is 05:00. Sinusoidal rate infusion schedule: start time is $00: 00$, completed time is $06: 00$, peak flow time is 03:00 (Figure 1).

\section{Evaluation criteria of efficacy and adverse reaction of chrono-chemotherapy and the treatment of adverse reaction}

\section{Evaluation criteria of efficacy and adverse reaction}

Efficacy evaluation is preferably recommended by reference to solid tumor efficacy evaluation criteria in RECIST (V1.1) (27,28); adverse reaction evaluation is preferably recommended by reference to chemotherapy adverse reaction severity evaluation criteria in CTCAE (V4.0) (29).

\section{Treatment of chrono-chemotherapy adverse reaction}

Compared with conventional chemotherapy, chronochemotherapy can significantly reduce the total incidence of adverse reactions and the incidence of serious adverse reactions of grade III-IV, especially to the reduction of blood adverse reactions (leukocytes and neutropenia) and adverse reactions of the digestive tract (nausea, vomiting, and oral mucositis), being more significantly. The principle of treatment of adverse reactions to chrono-chemotherapy is the same as that of conventional chemotherapy.

At present, the circadian clock and circadian rhythm have been the research hotspots in life sciences and medicine $(8,9,19)$, and its research results provide a solid foundation for chrono-chemotherapy. Meanwhile, the clinical practice also proves that chrono-chemotherapy for OSCC can reduce adverse reactions and increase the efficacy of drugs $(16,17,25,26)$ and value for clinical application. However, there are still many issues that need further research: (I) although the current general evidence that chronochemotherapy for OSCC can reduce adverse drug reactions and increase drug efficacy, there is still a lack of large-scale randomized multi-center controlled clinical trial data, and there is also a lack of further stratified analysis data under the premise of large samples, such as the effects of factors like gender, age, different stages of the tumor, different genetic backgrounds and environment on the chronochemotherapeutic effect; (II) due to the different factors, such as rest-activity phases, living habits, dietary patterns and work of individuals, will affect the circadian rhythm, and even in the same individual the circadian rhythm may change in the different stages of the disease, the circadian rhythm may change. Therefore, how to accurately measure the individualized circadian rhythm, and individualized or precise chrono-chemotherapy according to the different individual circadian rhythm will be the future development direction. This consensus will also be revised in time with the development of chrono-chemotherapy, to guide clinicians to standardize chronochemotherapy for OSCC timely and expertly.

\section{Acknowledgments}

Funding: This study was supported by the Natural Science Foundation of Chongqing, China (cstc2018jcyjA0481).

\section{Footnote}

Conflicts of Interest: All authors have completed the ICMJE 
uniform disclosure form (available at https://fomm. amegroups.com/article/view/10.21037/fomm.2019.11.01/ coif). JWZ serves as an unpaid editor-in-chief of Frontiers of Oral and Maxillofacial Medicine. The other authors have no conflicts of interest to declare.

Ethical Statement: The authors are accountable for all aspects of the work in ensuring that questions related to the accuracy or integrity of any part of the work are appropriately investigated and resolved.

Open Access Statement: This is an Open Access article distributed in accordance with the Creative Commons Attribution-NonCommercial-NoDerivs 4.0 International License (CC BY-NC-ND 4.0), which permits the noncommercial replication and distribution of the article with the strict proviso that no changes or edits are made and the original work is properly cited (including links to both the formal publication through the relevant DOI and the license). See: https://creativecommons.org/licenses/by-nc-nd/4.0/.

\section{References}

1. Siegel RL, Miller KD, Jemal A. Cancer statistics, 2018. CA Cancer J Clin 2018;68:7-30.

2. Pfister D, Spencer S, Adelstein D, et al. NCCN clinical practice guidelines in oncology (NCCN Guidelines $\left.{ }^{\circledR}\right)$ for head and neck cancers. Version 2.2018. Fort Washington: National Comprehensive Cancer Network (NCCN) [DB/OL]. 2018. Available online: https://www.nccn.org/ professionals/physician_gls/default.aspx

3. Posner MR, Hershock DM, Blajman CR, et al. Cisplatin and fluorouracil alone or with docetaxel in head and neck cancer. N Engl J Med 2007;357:1705-15.

4. Yanamoto S, Umeda M, Kioi M, et al. Multicenter retrospective study of cetuximab plus platinumbased chemotherapy for recurrent or metastatic oral squamous cell carcinoma. Cancer Chemother Pharmacol 2018;81:549-54.

5. Ballesta A, Innominato PF, Dallmann R, et al. Systems chronotherapeutics. Pharmacol Rev 2017;69:161-99.

6. Ortiz-Tudela E, Innominato PF, Rol MA, et al. Relevance of internal time and circadian robustness for cancer patients. BMC Cancer 2016;16:285.

7. Ozturk N, Ozturk D, Kavakli IH, et al. Molecular aspects of circadian pharmacology and relevance for cancer chronotherapy. Int J Mol Sci 2017. doi: 10.3390/ ijms18102168.
8. FitzGerald GA. Circadian Rhythms. Temporal targets of drug action. Science 2014;346:921-2.

9. Sehgal A. Physiology flies with time. Cell 2017;171:1232-5.

10. Turek FW. Circadian clocks: Not your grandfather's clock. Science 2016;354:992-3.

11. Innominato PF, Roche VP, Palesh OG, et al. The circadian timing system in clinical oncology. Ann Med 2014;46:191-207.

12. Innominato PF, Lévi FA, Bjarnason GA. Chronotherapy and the molecular clock: Clinical implications in oncology. Adv Drug Deliv Rev 2010;62:979-1001.

13. Ortiz-Tudela E, Mteyrek A, Ballesta A, et el. Cancer chronotherapeutics: experimental, theoretical, and clinical aspects. Handb Exp Pharmacol 2013;(217):261-88.

14. Lévi F, Okyar A, Dulong S, at el. Circadian timing in cancer treatments. Annu Rev Pharmacol Toxicol 2010;50:377-421.

15. Sancar A, Lindsey-Boltz LA, Gaddameedhi S, at el. Circadian clock, cancer, and chemotherapy. Biochemistry 2015;54:110-23.

16. Yang K, Chen R, Yu X. Neoadjuvant chrono-chemotherapy for the patients with oral squamous cell carcinoma. Journal of Practical Stomatology 2005;21:389-91.

17. Chen D, Cheng J, Yang K, et al. Retrospective analysis of chronomodulated chemotherapy versus conventional chemotherapy with paclitaxel, carboplatin, and 5 -fluorouracil in patients with recurrent and/or metastatic head and neck squamous cell carcinoma. Onco Targets Ther2013;6:1507-14.

18. Ye H, Yang K, Tan XM, et al. Daily rhythm variations of the clock gene PER1 and cancer-related genes during various stages of carcinogenesis in a golden hamster model of buccal mucosa carcinoma. Onco Targets Ther 2015;8:1419-26.

19. Callaway E, Ledford H. Medicine Nobel awarded for work on circadian clocks. Nature 2017;550:18.

20. Zhang R, Lahens NF, Ballance HI, et al. A circadian gene expression atlas in mammals: implications for biology and medicine. Proc Natl Acad Sci U S A 2014;111:16219-24.

21. Zhao N, Yang K, Tang K, et al. Chronchemotherapy of oxaliplatin to oral squamous cell carcinoma. Hua Xi Kou Qiang Yi Xue Za Zhi 2013;31:131-5

22. Zhao N, Tang H, Yang K, et al. Circadian rhythm characteristics of oral squamous cell carcinoma growth in an orthotopic xenograft model. Onco Targets Ther 2013;6:41-6.

23. Hori K, Zhang QH, Li HC, et al. Timing of cancer chemotherapy based on circadian variations in tumor 
tissue blood flow. Int J Cancer 1996;65:360-4.

24. Blumenthal RD, Osorio L, Ochakovskaya R, et al. Regulation of tumour drug delivery by blood flow chronobiology. Eur J Cancer 2000;36:1876-84.

25. Yang K, Zhang F, Li Y, et al. Clinical study of neoadjuvant chrono-chemotherapy for the elderly patients with oral squamous cell carcinoma. Chinese Journal of Geriatric Dentistry 2005;3:23-25

26. Tsuchiya Y, Ushijima K, Noguchi T, et al. Influence of a dosing-time on toxicities induced by docetaxel, cisplatin and 5-fluorouracil in patients with oral squamous cell carcinoma; a cross-over pilot study. Chronobiol Int 2018;35:289-94.

\section{doi: 10.21037/fomm.2019.11.01}

Cite this article as: Yang $\mathrm{K}$, Guo W, Sun M, Zheng J, Li L, Tang Z, Ren G, Chen D, Lv X, Zhang J, Han Z, Xi Q, Sun Z, Chen X, Zhang T, Che Z, Ran W, Meng J, Huang G, Tang H, $\mathrm{Xiao}$ C. Chinese expert consensus on chrono-chemotherapy for oral squamous cell carcinoma. Front Oral Maxillofac Med 2019;1:9.
27. Therasse P, Arbuck SG, Eisenhauer EA, et al. New guidelines to evaluate the response to treatment in solid tumors. European Organization for Research and Treatment of Cancer, National Cancer Institute of the United States, National Cancer Institute of Canada. J Natl Cancer Inst 2000;92:205-16.

28. Eisenhauer EA, Therasse P, Bogaerts J, et al. New response evaluation criteria in solid tumours: revised RECIST guideline (version 1.1). Eur J Cancer 2009;45:228-47.

29. HHS, NIH, NCI. Common Terminology Criteria for Adverse Events (CTCAE) Version 4.0[DB/OL].2009; Available online: http://evs.nci.nih.gov/ftp1/CTCAE/ About.html 